Journal of Advanced Research in Applied Mechanics

Journal homepage: www.akademiabaru.com/aram.html ISSN: 2289-7895

\title{
Intelligent Control for Ship Manoeuvering
}

\author{
Ahmed Yaseen Adnan ${ }^{1, *}$, Hannan Mohammed Abdul ${ }^{2}$, Mustaffa Kamal Iwan ${ }^{1}$ \\ Faculty of Marine Design Technology, Universiti Kula-Lumpur, Bandar Teknologi Maritim, Jalan Pantai Remis, 32200 Lumut, Perak, Malaysia \\ Faculty of Science, Agriculture and Engineering Newcastle University, UK (Singapore Unit), Singapore
}

\section{ABSTRACT}

In ship manoeuvering, the only two actuators are rudder and propeller revolution for the whole system. Therefore, by controlling these two for a designated purpose, automation in ship manoeuvering could be established. Classical control system like PID for track keeping or course changing has widely been used by on-board autopilot system. However, there are number of sophisticated ship manoeuveres, where the classical control systems fail and thus, the intelligent controllers are often encouraged to take over the classical systems. This paper is aimed to point out the application of Artificial Neural Network (ANN) controller for automatic ship berthing and Fuzzy Logic controller for waypoint controller in particular. Strategies as well as the formation of the two controllers are discussed briefly. In addition, simulations and experiments results are presented and compared to ensure the practical applicability of these intelligent controllers.

\section{Keywords:}

Intelligent controller, Artificial Neural

Network, Fuzzy Logic, Ship Berthing,

Waypoint controller

Copyright @ 2020 PENERBIT AKADEMIA BARU - All rights reserved

\section{Introduction}

Automation in ship operation is getting everybody's attention as the specialised knowledge of workers continues to decline. Apart from that, the increasing modern technologies often demand a promising solution of highly demanding control problems under increased uncertainty. In such situation, conventional approaches have been proposed for several control problems. However, successful applications can only be found within well-constrained environment and none is flexible enough to perform beyond its restricted zone. Consequently, numerous advancements have been made in developing intelligent systems. One of these is inspired by human's central nervous system called artificial neural network (ANN). This ANN consists of several interconnected simple non-linear system typically modelled by the transfer function therefore, has the capability to replicate human brains and perform the action that a human brain does in any particular situation. ANN as a controller was first initiated by Yamato et al., [1] for automatic ship berthing. Later on, Fujii et al., [2] confirmed its effectiveness as a controller for both supervised and non-supervised learning system especially

\footnotetext{
* Corresponding author.

E-mail address: yaseen.ahmed@unikl.edu.my
}

https://doi.org/10.37934/aram.67.1.19 
for AUVs. After him, ANN had been tried as a controller in different controlling aspects like temperature control, paper mill waste water treatment control, engine air/fuel ratio control, process control, arc welding control etc. In the field of berthing, after Hasegawa et al., [3] and Im et al., [4,5] had continued the research. Hasegawa proposed ANN combined with expert system to assist ANN, while Im proposed separate controllers instead of centralised one for command rudder and propeller revolution output respectively. Both proposals played a vital role individually for further development of this research, but in the presence of wind disturbances, ANN often failed to guide the ship successfully to the pier. In the meanwhile, Ohtsu et al. [6] proposed a new minimum time ship manoeuvring method using nonlinear programing method which allowed the user to set the desired equality and non-equality constraints for any type of ship manoeuvre. Taking the advantages of this method and using the concept of aircraft landing, for the first time a way of creating automated teaching data was proposed by Ahmed et al., [7] one of the authors of this paper. This concept not only ensured the consistency in teaching data for better learning of ANN but also raised the necessity of having ANN-PD controller rather than ANN alone for low speed course keeping stage under wind disturbances. This paper overviews on the said concept with its application in real time ship berthing operation.

In addition, this paper also mentions about waypoint tracking control problem which is basically how making the ship follow a given set of waypoints by controlling the rudder; Fossen [8] and Petterson [9]. There are several guidance algorithms exist as mentioned by Jensen [10], like pure pursuit guidance algorithm, Line-of-sight (LOS) guidance algorithms, etc. But, in both cases way point (WP) switching algorithm is necessary. Comparing to such existing algorithm, this paper describes a fuzzy based guidance algorithm which measures the nearness of next and second next waypoint one at a time and decides the desired course. In order to measure the nearness of WPs, distance to closest point approach (DCPA) and time to closest point approach (TCPA) are used, which are discussed at the later part of this paper. Then, as a course keeping controller, PD is used to match the desired heading. Therefore, basically the proposed controller has two control loops. The outer control loop belongs to the fuzzy that generates the desired course based on given waypoints for the inner control loop and the inner control loop makes the ship move towards the direction of minimising the heading error by controlling the rudder angle. In such controller, when the ship is away from the second next waypoint, the command course will consider only for the next waypoint. However, with the increase of nearness to the next waypoint, the course will modify by considering both next and second next waypoint. A brief discussion and some experiment results using this controller are included in this paper.

\section{Mathematical Model for Manoeuvring Prediction}

There are lot of vessels travelling all over the world equipped with a single rudder and a single screw propeller. 'Esso Osaka' is among them. The $3 \mathrm{~m}$ model of the ship which is scaled by 1:108.33 is chosen for this study. Availability of large amounts of captive model test results is one of few reasons of choosing this vessel. Its details are given in Table 1. 


\section{Table 1}

Principal particulars and parameters of model Ship

\begin{tabular}{cccccc}
\hline Hull & \multicolumn{3}{c}{ Rudder } \\
$L(\mathrm{~m})$ & 3 & $D_{p}(\mathrm{~m})$ & 0.084 & $b(\mathrm{~m})$ & 0.083 \\
$B(\mathrm{~m})$ & 0.48 & $P(\mathrm{~m})$ & 0.06 & $h(\mathrm{~m})$ & 0.1279 \\
$D(\mathrm{~m})$ & 0.2 & Pitch Ratio & 0.7151 & $A_{R}\left(\mathrm{~m}^{2}\right)$ & 0.0106 \\
$C_{b}$ & 0.831 & $Z$ & 5 & $\Lambda$ & 1.539 \\
\hline
\end{tabular}

The coordinate system used to formulate the equation of motion is shown in Figure 1 . Here, the ship heading is assumed as clockwise and wind direction as anti-clock wise positive.

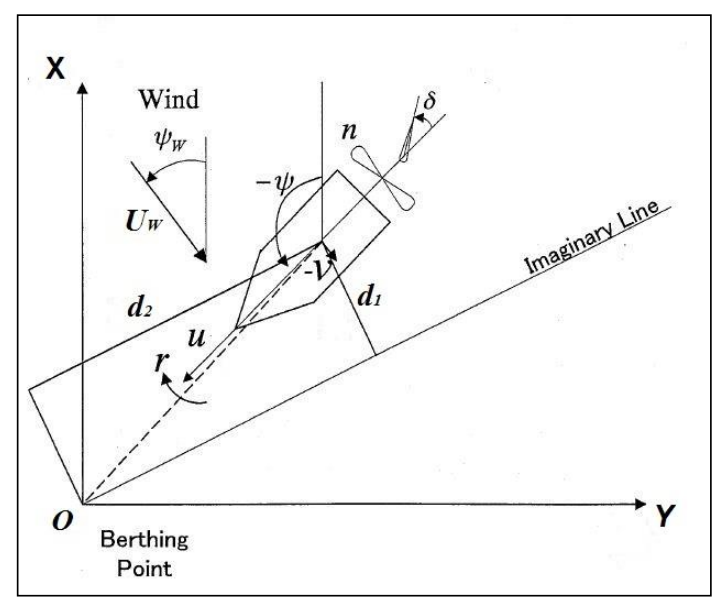

Fig. 1. Coordinate System

A modified version of mathematical model based on MMG [11] for describing the ship hydrodynamics in three degrees of freedom is used for this model ship. In the MMG model, not only hull, propeller and rudder forces are considered separately, but their interactions are also taken into account. The corresponding equations of motions at CG (centre of gravity) of the ship are expressed in Equation 1.

$$
\begin{aligned}
& \left(m+m_{x}\right) \dot{u}-\left(m+m_{y}\right) v r=X_{H}+X_{P}+X_{R}+X_{W} \\
& \left(m+m_{y}\right) \dot{v}+\left(m+m_{x}\right) u r=Y_{H}+Y_{P}+Y_{R}+Y_{W} \\
& \left(I_{Z Z}+J_{Z Z}\right) \dot{r}=N_{H}+N_{P}+N_{R}+N_{W}
\end{aligned}
$$

where, $X_{H}, Y_{H}, N_{H}$ : Hydrodynamic forces and moment acting on hull; $X_{P}, Y_{P}, N_{P}$ : Hydrodynamic forces and moment due to propeller; $X_{R}, Y_{R}, N_{R}$ : Hydrodynamic forces and moment due to rudder; $X_{W}, Y_{W}, N_{W}$ : Hydrodynamic forces and moment due to wind. In addition, Fujiwara wind model [12] is adopted to create the wind disturbances and instead of steady wind, gust wind is adopted (Davenport [13]). 


\section{Neural Network Controller and its Training for Ship Berthing}

In order to train neural network, consistency in teaching data is of prime concern. To ensure that, this paper describes how the aircraft landing procedure is adopted for ship berthing. First and foremost, the berthing manoeuvre is planned for course changing to meet a final desired ship heading. This final heading with no sway speed and angular velocity will align the ship to a reference line known as imaginary line that will act as a runway for further descent according to the speed response equation as proposed by Endo et al., [14]. The imaginary line is assumed here to be $15 \mathrm{~L}$ of length according to the IMO standard and 30 을 with the pier. The berthing goal point is also considered at a distance of $1.5 \mathrm{~L}$ from the actual pier to ensure safety.

\subsection{Teaching Data Creation}

Nonlinear programming language (NPL) method for minimum time course changing manoeuvre is utilised to create the consistent teaching data for course changing manoeuvre and the repeated optimisation technique as explained by Ahmed and Hasegawa [7] is used to get several course changing trajectories for ship's different initial heading to one particular final heading which is 240응 with no sway and angular velocity. Here, the final heading 240 o means making an angle 30 with the pier i.e. the ship will align with the imaginary line after course changing.

After course changing, the ship is expected to go straight with no sway velocity and yaw rate. But, in reality slight wind disturbances while reducing speed may cause drastic course change as the rudder becomes gradually ineffective with relatively low water inflow velocity. Considering the difficulties in maintaining the course in such situation, this paper highlights a PD controller mentioned in the Equation 2 to use for course keeping. The coefficients used for the controller are tuned on a trial basis to ensure earlier response of the controller in any slight disturbances. The sequence of telegraph maintained here is half ahead during course changing, then it is followed by show ahead, dead slow ahead, engine idling and at last propeller reversing. To judge the proper timing of telegraph order without damaging the engine and propeller shaft, a time constant Tp is used which is mentioned in Equation 3.

$$
\begin{aligned}
& \delta_{\text {order }}=C_{1} *\left(\psi_{d}-\psi\right)-C_{2} * \dot{\psi}-C_{3} * d_{1} \\
& \Rightarrow\left\{\begin{array}{l}
\text { if } \delta_{\text {order }}>0^{0}, \delta_{\text {order }}=10^{0} \\
\text { if } \delta_{\text {order }}=0^{0}, \delta_{\text {order }}=0^{0} \\
\text { if } \delta_{\text {order }}<0^{0}, \delta_{\text {order }}=-10^{0}
\end{array}\right\} \\
& T_{P} \frac{d U(t)}{d t}+U(t)=K_{P} n(t)
\end{aligned}
$$

where, $\psi d$ : desired heading; $\psi$ : current heading; $\psi$ : yaw rate; $d 1$ : a deviation from the imaginary line; $C 1 \sim C 3$ : coefficients; $U(t)$ : Ship velocity $(\mathrm{m} / \mathrm{s}) ; n(t)$ : Propeller revolution $(\mathrm{rps}) ; T_{p}$ : Time constant; $K_{p}:$ Gain.

Finally, by adding these course change and course keeping data, the total teaching data is formed as shown in Figure 2(a). 


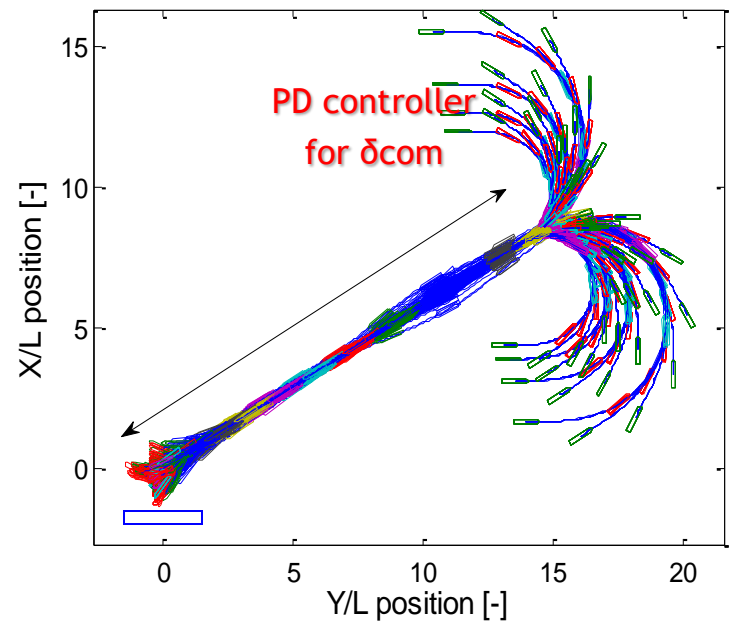

(a)

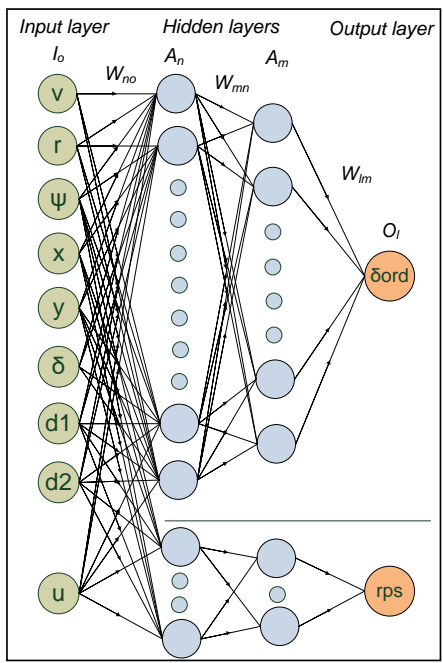

(b)

Fig. 2. (a) Complete teaching data; (b) ANN controller

\subsection{Training Neural Network}

Two separate networks are used instead of centralised controller for rudder and propeller revolution outputs respectively. In addition, two hidden layers are introduced for learning complex relationship among inputs and output. Neurons in each hidden layer are determined by ensuring minimum mean squared error (MSE) value after learning. Finally, Lavenberg-Marquardt algorithm is used as training function. Figure 2(b) illustrates the ANN controller formed for the berthing purpose.

\section{Results for Ship Berthing}

Sheer number of combinations of ship's initial heading and starting point are possible to judge the robustness of the proposed controller. Left side of the Figure 3 shows ship with three different headings tested for the same starting point. In case of initial heading 160 , the ANN decides to take a port turn first and then it starts its expected starboard turn gradually. Therefore, the ship follows a long way for course changing and there exists a large gap between the ship and imaginary line. However, the PD controller works effectively to minimise such existing gap and at last, the ship successfully stops within the expected zone. For the other two cases, the ANN controller takes proper decision and after a slight port turn, the ship starts its starboard turn as expected. The simulations are done under similar wind disturbances which is average wind velocity of $1.5 \mathrm{~m} / \mathrm{s}$ from 315 o wind direction. Right side of Figure 3 demonstrates the situation for ship starting with the same initial heading, but from three different arbitrary points. In all three cases, the controllers take different decision based on surroundings and succeeded to guide the ship up to the desired safety zone. In case 1, the controller also increases the propeller revolution during idling stage that is similar to boosting phenomenon. The wind disturbances considered in all three cases are the same, which is average wind velocity of $1.5 \mathrm{~m} / \mathrm{s}$ from 180 ․ wind direction. 


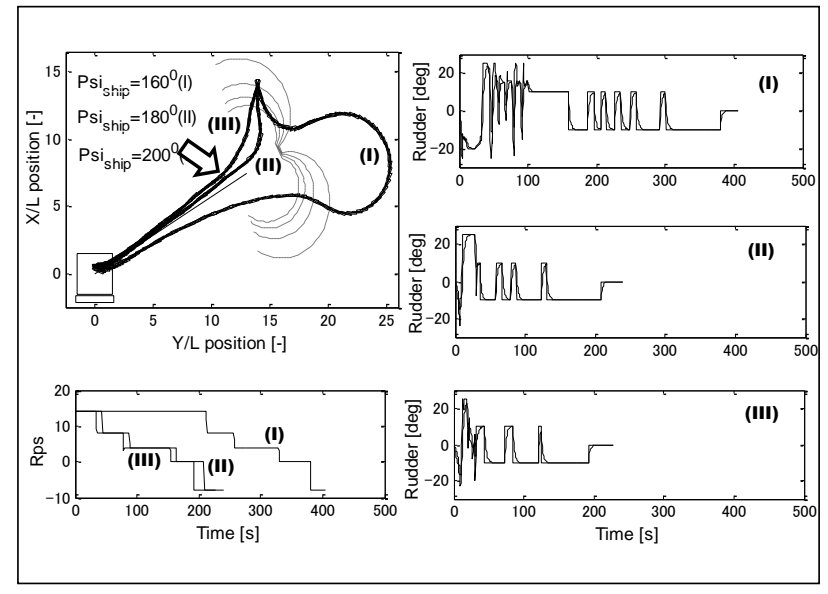

(a)

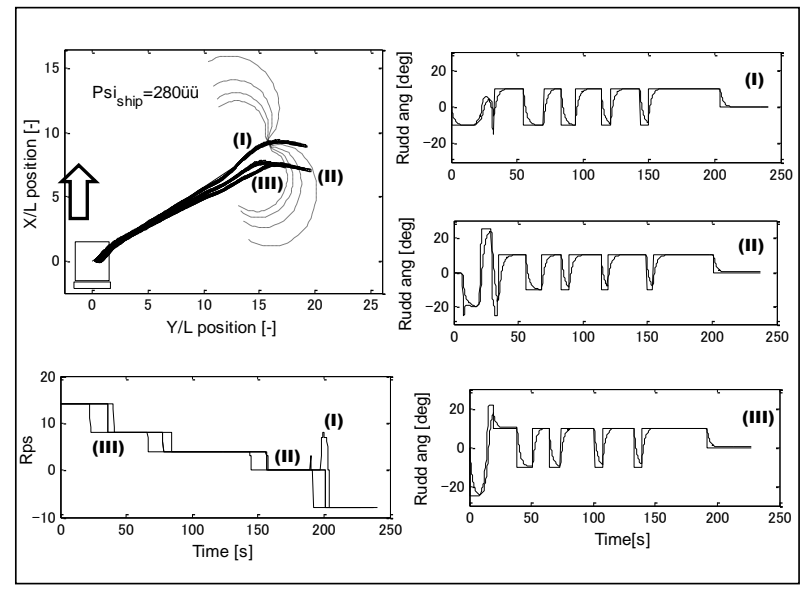

(b)

Fig. 3. (a) Simulation results for LHS approach (b) RHS approach

Experiments are conducted by utilizing the free running experiment system for Esso Osaka model ship. Both LHS and RHS approaches are investigated and the controllers are found suitable enough to guide the properly with in the desired zone with surge velocity less than $0.05 \mathrm{~m} / \mathrm{s}$. Figures 4 represents such experiment results. Here, the controller behaves differently depending on different initial conditions and wind disturbances.

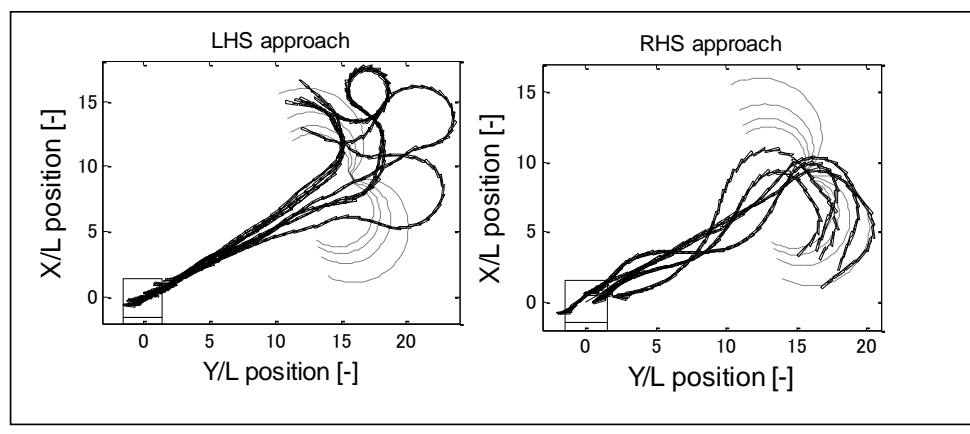

(a)

(b)

Fig. 4. (a) Experiment results for LHS approach (b) RHS approach

\section{Fuzzy Controller for Way Point Control}

Fuzzy controller is an alternative solution of variety of nonlinear control problems. Optimal control laws can be implemented based on ship operator's knowledge while making the fuzzy control rules. This paper focuses on the fuzzy reasoned waypoint controller to guide the ship from its current state to the given set points. Here, the navigation path planning is done based on the given set points called waypoints (WP) to be passed. Near the turning point, the fuzzy reasoning system will decide to choose the appropriate course defined by the next two WPs and after deciding the appropriate course by fuzzy reasoning, the course is corrected using a PD controller. So it is a double loop control scheme as shown in Figure 5. For the outer loop, fuzzy controller is used to feed the desired heading to the inner loop after getting feedback of the ship's position. On the other hand, PD controller is used in the inner loop to keep that desired course. 


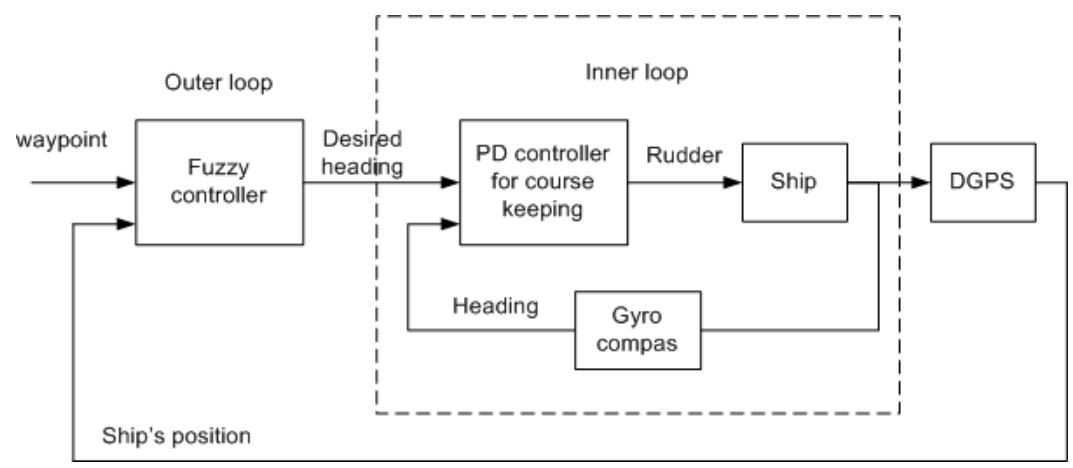

Fig. 5. Control loop

\subsection{Controller Design}

Fuzzy control laws similar to collision avoidance is used. However, instead of collision risk, nearness is reasoned. Near the turning point, the fuzzy system will decide to choose the appropriate course defined by the next two WPs as given in Equation 4 and shown in Figure 6.

$$
\psi_{I}=\psi_{1}+\left(\psi_{2}-\psi_{1}\right) * C D H
$$

where, $\psi_{I}$ is order of course change, $\psi_{1}$ is course of the shortest path to the next WP, $\psi_{2}$ is course of the shortest path to the second next WP and $C D H$ is the reference degree to the second next WP $(0 \leq C D H \leq 1)$, calculated by fuzzy.

To judge the nearness of the waypoint, TCPA (time to closest point of approach) and DCPA (distance of the closest point of approach) are used for fuzzy reasoning. Figure 7 describes the bearing relationship between the ship and waypoint.

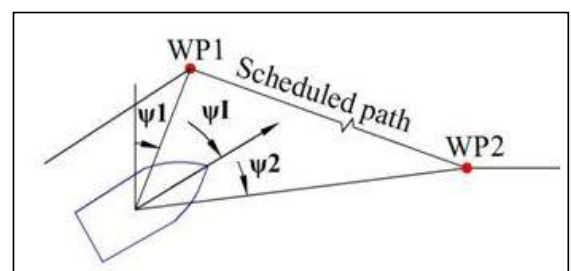

Fig. 6. Course command near a course changing point

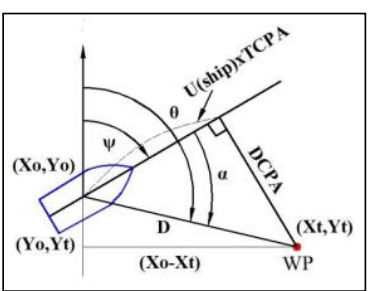

Fig. 7. Bearing relation between ship and waypoint

Here, $\psi$ : ship's heading; $\theta$ : encountering angle of way point from vertical axis; $\alpha$ : bearing angle of waypoint from the ship. If the value of $\psi, \theta$ or $\alpha$ becomes negative, then $2 \pi$ is added to make to them positive.

The fuzzy rules used here are similar to collision avoidance, i.e. "if DCPA is very short and TCPA is also very short, then $C D H$ is very big". It means, if the ship is very far from second next waypoint, the command course will consider only for the next waypoint. However, with the increase of nearness, the command course will be modified according to both next and second next waypoint. During the navigational path planning, the switching of waypoints is determined by TCPA value. Negative value of it for a particular waypoint ensures the ship already exceeded that point. Therefore, the controller considers the next pair of waypoint for further approach. After deciding the appropriate course by 
fuzzy reasoning, the course is corrected using a PD controller as shown in Equation 5 to correct the heading.

$$
\begin{aligned}
& \delta_{\text {order }}=K_{p}\left(\psi_{I}-\psi\right)-K_{d} \dot{\psi} \\
& \Rightarrow \text { if }\left\{\begin{array}{l}
\delta_{\text {order }} \geq 25^{0}, \delta_{\text {order }}=25^{0} \\
\delta_{\text {order }} \leq-25^{0}, \delta_{\text {order }}=-25^{0}
\end{array}\right.
\end{aligned}
$$

where, $\psi_{I}$ : desired heading calculated by fuzzy reasoning; $\psi$ : ship's current heading; $\dot{\psi}:$ the yaw rate; $K_{P}$ : proportional gain; $K_{D}$ : differential gain.

\section{Results for Waypoint Controlling}

Simulations are done using the fuzzy controller for different sets of waypoint including both port and starboard turn. Gust wind from different directions is also tested to judge the effectiveness of the control under wind disturbances. Moreover, waypoints are set for ' $\mathrm{S}$ ' letter shape and Figure $8(\mathrm{a})$ illustrates such result. Here, the simulation is done under wind of $1.5 \mathrm{~m} / \mathrm{s}$ from 0 . The result seems quite promising and the resulting a trajectory almost matches with the set alphabet shape.

Experiments are also done for Esso Osaka model ship and one of the corresponding results is shown in Figure 8 (b) where the waypoints that are set on the left side of the ship. Firstly, the controller started with relatively large port rudder and then followed by starboard rudder to adjust the existing yaw rate to guide it for the next pair of waypoint. It is evident that, the ship almost passes through the given set points.

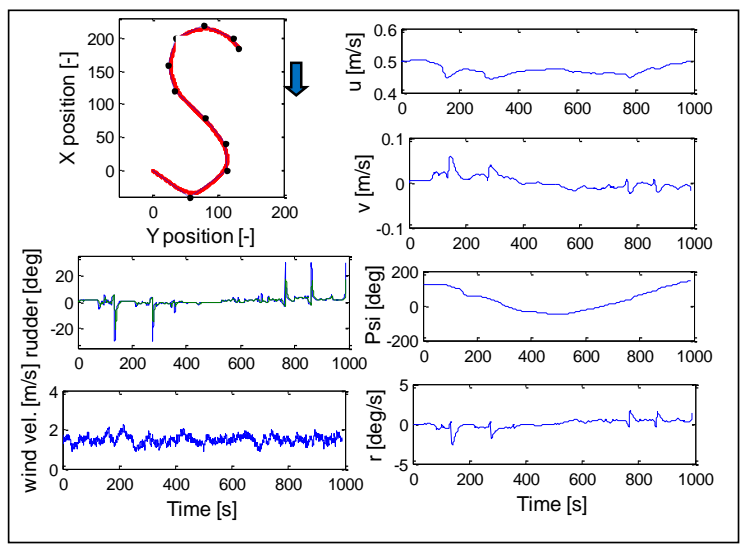

(a)

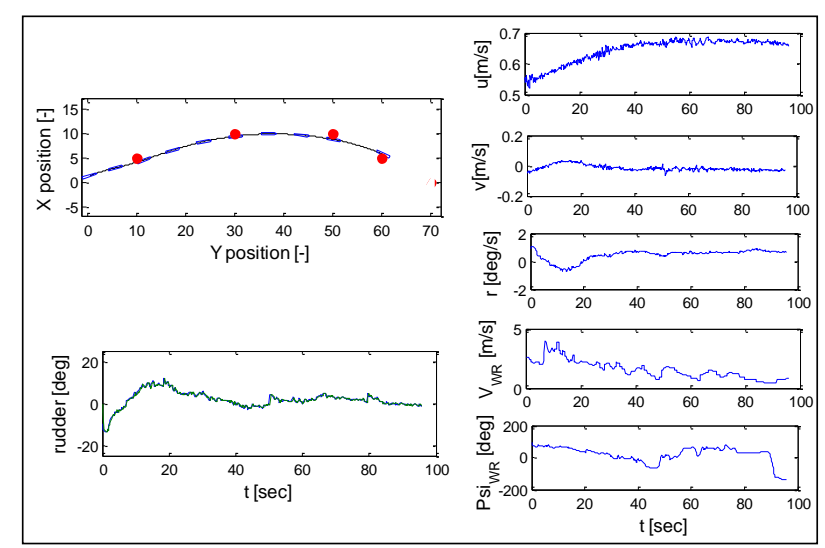

(b)

Fig. 8. (a) Simulation result; (b) Experiment result

\section{Conclusions}

This paper mentions about the application of intelligent controllers for two different types of ship manoeuvring which are ship berthing and waypoint controlling. The first part of this paper focuses on an optimisation technique to create consistent teaching data for training a multi-layered ANN controller. Following the control strategy, several simulations are done to judge the robustness of the controller under gust wind disturbances and found successful. Experiments are also carried out 
for the model ship by utilizing the free running experiment system. While performing the experiments, the controller has found to behave in some particular ways depending on different initial conditions and wind disturbances. However, it manages to guide the ship successfully within the desired stopping zone.

The later part of this paper presents a double loop feedback controller for waypoint navigation. The outer loop belongs to fuzzy that generates the desired course for the inner loop for necessary course correction. Based on the human operator's manipulating experience, the nearness of the next waypoint is measured and the reference degree to the second next waypoint is modified by fuzzy. In the meantime, if the TCPA becomes negative for the waypoint, next consecutive pair is considered for further navigation. Such control scheme is then used for simulations and experiments for different sets of waypoint and the corresponding results are found quite promising.

To conclude, each controller has its own application zone in which it acts its best. Therefore, to select a suitable controller for best result has always been challenging. Experience and nature of input-output relation will decide whether conventional or intelligent controller suits best for a given plant.

\section{REFERENCES}

[1] Yamato, H., Uetsuki, H. and Koyama, T. 1990. Automatic Berthing by Neural Controller. Proc. Of Ninth Ship Control Systems Symposium, 3, 183-201.

[2] Fujii, Teruo, and Tamaki Ura. "Neural-network-based adaptive control systems for AUVs." Engineering Applications of Artificial Intelligence 4, no. 4 (1991): 309-318. https://doi.org/10.1016/0952-1976(91)90045-8

[3] Hasegawa, K. "Automatic Berthing Control System Using Network and Knowledgebase." Journal of the Society of Naval Architects of Japan 220 (1993): 135-143.

[4] Im, Namkyun, and Kazuhiko Hasegawa. "A study on automatic ship berthing using parallel neural controller (2nd Report)." Journal of the Kansai Society of Naval Architects, Japan 2002, no. 237 (2002): 237_127-237_132.

[5] Im, Namkyun, and Kazuhiko Hasegawa. "A study on automatic ship berthing using parallel neural controller (2nd Report)." Journal of the Kansai Society of Naval Architects, Japan 2002, no. 237 (2002): 237_127-237_132.

[6] Mizuno, Naoki, Masaki Kuroda, Tadatsugi Okazaki, and Kohei Ohtsu. "Minimum time ship maneuvering method using neural network and nonlinear model predictive compensator." Control Engineering Practice 15, no. 6 (2007): 757-765.

https://doi.org/10.1016/i.conengprac.2007.01.002

[7] Ahmed, Yaseen Adnan, and Kazuhiko Hasegawa. "Automatic ship berthing using artificial neural network trained by consistent teaching data using nonlinear programming method." Engineering applications of artificial intelligence 26, no. 10 (2013): 2287-2304. https://doi.org/10.1016/j.engappai.2013.08.009

[8] Fossen, Thor I. Guidance and control of ocean vehicles. Vol. 199, no. 4. New York: Wiley, 1994.

[9] Pettersen, Kristin Y., and Even Lefeber. "Way-point tracking control of ships." In Proceedings of the 4Oth IEEE Conference on Decision and Control (Cat. No. 01CH37228), vol. 1, pp. 940-945. IEEE, 2001.

[10] Jensen, Tor Marius. "Waypoint-following guidance based on feasibility algorithms." Master's thesis, Institutt for teknisk kybernetikk, 2011.

[11] Kobayashi, H., J. J. Blok, R. Barr, Y. S. Kim, and J. Nowicki. "Specialist Committee on Esso Osaka: Final Report and Recommendations to the 23rd ITTC,"." In Proceedings of the 23rd International Towing Tank Conference, pp. 8-14. 2003.

[12] Fujiwara, Toshifumi, Michio Ueno, and Tadashi Nimura. "Estimation of wind forces and moments acting on ships." Journal of the Society of Naval Architects of Japan 1998, no. 183 (1998): 77-90. https://doi.org/10.2534/ijasnaoe1968.1998.77

[13] Davenport A.G. (1967). The dependence wind loads on meteorological parameters. Proc. of conference on wind effects on buildings and structures.

[14] Endo, Makoto, and Kazuhiko Hasegawa. "Passage planning system for small inland vessels based on standard paradigms and manoeuvres of experts." MARSIM'03 (2003). 